\title{
QUANTUM HALL FRACTIONS IN ULTRACOLD ATOMIC VAPORS
}

\author{
N. Regnault and Th. Jolicour \\ Laboratoire Pierre Aigrain, Département de Physique, \\ 24, rue Lhomond, 75005 Paris, France \\ Nicolas.Regnault@lpa.ens.fr, Thierry.Jolicoeur@lpa.ens.fr
}

\begin{abstract}
Atomic vapors can be prepared and manipulated at very low densities and temperatures. When they are rotating, they can reach a quantum Hall regime in which there should be manifestations of the fractional quantum Hall effect. We discuss the appearance of the principal sequence of fractions $\nu=p /(p \pm 1)$ for bosonic atoms. The termination point of this series is the paired Moore-Read Pfaffian state. Exotic states fill the gap between the paired state and the vortex lattice expected at high filling of the lowest Landau level. In fermionic vapors, the $p$-wave scattering typical of ultralow energy collisions leads to the hard-core model when restricted to the lowest Landau level.
\end{abstract}

PACS numbers: 03.75Kk, 05.30.Jp, 73.43.Cd, 73.43.Lp

\section{INTRODUCTION}

It is now feasible to study atomic vapors, fermionic as well as bosonic, in magneto-optical traps at very low temperature and in a dilute regime. Progress in the trapping and cooling of atoms has led to the observation of the Bose condensation of many elements, notably all alkalis. This regime can be studied with exquisite precision since it can be treated by the mean-field theory for bosons, i.e. the Gross-Pitaevskii equation, something which was not available for the dense, strongly interacting, liquid Helium-4. Similarly fermionic vapors can be cooled down to the quantum degenerate limit where the Fermi sea becomes dominant. In the Bose case, the cooling can be performed efficiently for a single hyperfine species. In the case of fermions, when there is a single hyperfine species, the ultralow energy scattering takes place in the partial wave $L=1$ leading to much weaker interactions. As a consequence, it is more difficult to cool down fermions. One escape is the sympathetic cooling involving two hyperfine species.

The cold atoms display a wealth of interesting phenomena when the trap is rotating 1 , 2, 3, 4]. In the Bose case, the condensed atoms are in a superfluid state and as such, they have a very special response to rotation. If the rotation is slow enough the flow is irrotational but beyond a critical value of the rotation velocity there is nucleation of a vortex in which essentially all atoms occupy a state with unit angular momentum along the axis of rotation. Beyond this regime one creates more vortices and their mutual repulsion leads to the formation of the celebrated Abrikosov triangular lattice. This physics takes place when the angular momentum (in units of $\hbar$ ) is of the order of the number of particles. For even faster rotations, it has been remarked that one should enter a quantum Hall regime [5]. This takes place when the angular momentum is of the order of the square of the number of particles. Such a regime is reached when the centrifugal force exactly compensates the harmonic trapping force confining the atoms and then one is left only with the Coriolis force which is formally analogous to a magnetic field. If the confinement along the rotation axis is strong enough, then the gas is in a two-dimensional (2D) regime and this is the arena of the fractional quantum Hall effect (FQHE). Present experiments have already reached the required fast rotation limit but it remains to create a truly $2 \mathrm{D}$ regime and to achieve temperatures low enough to concentrate all atoms in the lowest Landau level (LLL). The huge degeneracy of the Landau level problem is then lifted only by interactions, the s-wave collisions in the case of bosons.

Rotation is also an interesting probe of the physics of fermions. Notably it has been suggested that it may reveal the BCS condensation expected for attractive interactions. As in the case of bosons, the condensed state is a superfluid with reduced momentum of inertia and in the irrotational flow regime there are signatures of superfluidity in the quadrupolar modes of oscillations of the atomic cloud. These signatures have been observed in the case of the Bose condensates [6].

In the fast rotation regime, fermions also will reach the quantum Hall regime. The main novelty with respect to condensed matter physics is now the nature of interactions between the atoms [7]. When there is a single hyperfine species then collisions are p-wave in the low-energy limit : colliding pairs of atoms have relative angular momentum $L=1$. When projected in the lowest landau level, this is exactly the so-called hard-core model [8], well known from the very beginning of the studies of the FQHE. For this interaction, the celebrated Laughlin wavefunction for fermions is the exact ground state of the system for a special value of the angular momentum. Many (if not all) of the features of the FQHE in solid-state devices should thus be accessible to experiments in this regime. Notably there should be an emergent Fermi sea for half-filling of the LLL as a termination point of the FQHE series of fractions $\nu=p /(2 p \pm 1)$ to be contrasted with the gapped paired state of bosons at $\nu=1$.

In section 2 , we discuss the basics of the rotating frame formalism. In section 3 , we explain the relevant parameters 
of atomic collisions. Section 4 is devoted to the study of FQHE fractions for bosons. Section 5 discusses the special case of complete filling of the lowest Landau level by the bosons. Section 6 discusses the transition towards the vortex lattice. Section 7 is devoted to the case of trapped fermions and their FQHE physics. Finally section 8 contains our conclusions.

\section{TRAPPED ATOMS IN ROTATION}

Transformation to the rotating frame can be done by the substitution $\mathcal{H} \rightarrow \mathcal{H}-\omega L_{z}$ where $L_{z}$ is the total angular momentum along the rotation axis. In the rotating frame [9], the Hamiltonian describing $\mathrm{N}$ trapped atoms of mass $m$ is given by :

$$
\begin{aligned}
\mathcal{H} & =\sum_{i=1}^{N} \frac{1}{2 m}\left(\mathbf{p}_{i}-m \omega \hat{\mathbf{z}} \times \mathbf{r}_{i}\right)^{2}+\frac{1}{2} m\left(\omega_{0}^{2}-\omega^{2}\right)\left(x_{i}^{2}+y_{i}^{2}\right) \\
& +\frac{1}{2} m \omega_{z}^{2} z_{i}^{2}+\sum_{i<j}^{N} V\left(\mathbf{r}_{i}-\mathbf{r}_{j}\right) .
\end{aligned}
$$

Here we have assumed a harmonic trapping potential in the $x y$ plane with $x y$ trap frequency $\omega_{0}$. There is also a trapping potential along the z-axis with frequency $\omega_{z}$ and the angular velocity vector is $\omega \hat{\mathbf{z}}$. For $\omega$ close to $\omega_{0}$, the physics is that of charge-e particles in a magnetic field $\mathbf{B}=(2 m \omega / e) \hat{\mathbf{z}}$, corresponding to a magnetic length $\ell=\sqrt{\hbar /(2 m \omega)}$. The cyclotron frequency of the equivalent magnetic problem is thus $\omega_{c}=2 \omega$. Typically, the trap frequencies are in the range 10-100 Hz. Condensates may be prepared in very anisotropic potentials, one or twodimensional. The quantum Hall states will apppear only in an effectively two-dimensional set-up. This may be achieved by use of an optical lattice along the $z$-axis. For the typical traps, when rotated at the critical frequency, the Landau level spacing $\hbar \omega_{c}$ is of the order of a few nanoKelvins. Again the quantum Hall phases will require temperatures below this value to exist.

Present studies of the vortex lattice that forms at low rotation frequencies use an additional potential which is anisotropic in the $x y$ plane to stir the condensate up to the required angular momentum. Once the required regime is reached one can then remove the stirring potential, going back to full rotational symmetry, and hope that thermodynamic equilibrium is reached in the rotating frame after some relaxation time. To go beyond the critical value for the rotation frequency, one has to add an extra confining potential to prevent explosion of the gas, e.g. a quartic term is feasible. It is important to note that one has in general knowledge neither of the rotation frequency of the gas nor of its angular momentum. These crucial quantities have to be measured directly or inferred from related quantities.

\section{COLLISIONS AND PSEUDOPOTENTIALS}

In the context of ultracold trapped gases, it is important to note that the collisions take place with a characteristic energy scale which is set by the temperature, nanoKelvins or less, and many orders of magnitude less than the energy scales of the internal degrees of freedom of the interatomic potential. In this limit, the scattering problem simplifies tremendously and is given by only a few partial waves. For a single species of bosons, the scattering takes places only for zero relative angular momentum and the phase shift may be written as $\delta_{0}(k) \simeq-k a_{s}$ for $k \rightarrow 0$, where $k$ is the momentum and $a_{s}$ is the s-wave scattering length. This limiting case may be studied by use of the so-called pseudopotential :

$$
V_{0}(\mathbf{r})=\left(4 \pi \hbar^{2} a_{s} / m\right) \delta^{(3)}(\mathbf{r})
$$

where $m$ is the mass of the bosonic atoms. This pseudopotential is known to lead to an ill-defined scattering problem in general and should be regularized in some cases. However it has the virtue of reproducing correctly the Born approximation of the true scattering problem. In the case of the fractional quantum Hall effect, we need only to diagonalize the interaction problem restricted to the lowest Landau level for many of the important questions. Since this is essentially first-order perturbation theory on a massively degenerate level, it is enough to use the singular unregularized potential Eq.(2).

In the case of a single species of fermions, the antisymmetry of the wavefunction implies that the scattering now takes place for relative angular momentum $L=1$. The relevant phase shift is now $\delta_{1}(k) \simeq-k^{3} a_{p}^{3} / 3$ for $k \rightarrow 0$, where 
$a_{p}$ is the p-wave scattering length. There is also a pseudopotential in the p-wave case :

$$
\hat{V}_{1}=\left(12 \pi a_{p}^{3} / m\right) \hat{\mathbf{p}} \delta^{(3)}(\mathbf{r}) \hat{\mathbf{p}},
$$

where $\hat{\mathbf{p}}$ is the tridimensional impulsion operator. If there are more than one hyperfine species then again s-wave scattering is allowed and thus dominates because of the stronger vanishing of the p-wave phase shift. The smallness of p-wave interactions is generally considered as a nuisance when cooling fermions. However it has recently been demonstrated [10] that it is possible to tune the scattering length by going through a resonance as a function of an applied external field. This is similar to the manipulation of the s-wave scattering by use of a Feshbach resonance. To obtain a sizeable FQHE gap in a Fermi gas, it may necessary to enhance the interactions by such a resonance.

\section{THE PRINCIPAL SEQUENCE}

We now discuss the FQHE physics for a single component Bose gas. If we are in a $2 \mathrm{D}$ regime at the critical frequency defined in Eq.(11) with the potential given by the Eq.(2), then we are exactly in the conditions required for the apparition of the FQHE for bosons with delta interactions. To make progress, it is possible to write down the wavefunctions and the hierarchical constructions invented for fermions and it is also possible to perform exact diagonalizations on small systems. Pioneering studies have been performed in refs.(11, 12, 13]). We have also followed these two strategies [14, 15]. In the numerical studies we used the spherical geometry which has been shown to be very useful in the case of fermions with Coulomb interactions [16]. Strictly speaking, the experiments most closely correspond to the geometry of an unbounded disk and it is thus important to translate our results also in this set-up. In the symmetric gauge and in the unbounded plane, the eigenstates of the lowest Landau level are given by :

$$
\phi_{m}(z)=\frac{1}{\sqrt{\ell \sqrt{\pi}}} z^{m} \mathrm{e}^{-|z|^{2} / 4 \ell^{2}},
$$

where we use the complex notation $z=x+i y$ and $m$ is the angular momentum of the state along $z$. To reside entirely in the lowest Landau level, an otherwise arbitrary quantum state should be of the form :

$$
\Psi\left(z_{i}\right)=f\left(z_{i}\right) \mathrm{e}^{-\sum_{i}\left|z_{i}\right|^{2} / 4 \ell^{2}},
$$

where $f$ is an analytic function of the $z_{i}$ 's.

\section{A. The $\nu=1 / 2$ Laughlin state}

The Laughlin wavefunction for bosons is given by :

$$
\Psi^{(m=2)}=\prod_{i<j}\left(z_{i}-z_{j}\right)^{2} \mathrm{e}^{-\sum_{i}\left|z_{i}\right|^{2} / 4 \ell^{2}} .
$$

This state is in the LLL and is an exact zero-energy eigenstate of the delta interaction [5]. It is the lowest total angular momentum state with these properties. For higher angular momentum, it is always possible to multiply $\Psi^{(m=2)}$ by an arbitrary symmetric polynomial in the $z_{i}$ 's to get more zero-energy states. These states are edge excitations at least for not too high momentum. The state Eq.(6) describes a droplet of incompressible fluid centered at the origin and the mean density corresponds to a filling fraction $\nu=1 / 2$ of the LLL. More generally the filling fraction appears in the relation between the number of particles and the total angular momentum : $L_{z}=N^{2} /(2 \nu)$ for large $N$ and $L_{z}$. A sample spectrum for $\mathrm{N}=5$ bosons is displayed in Fig.(1). The ground states are the Laughlin states and the edge excitations. For larger angular momentum, there is also the quasihole excitation which is gapless, a peculiarity of the delta interaction. The Laughlin fluid also support quasielectron excitations obtained by reducing the angular momentum by $\mathrm{N}$ unit exactly : this is the first nonzero energy state in Fig. (1). It has descendants with same energy due to the center-of-mass motion (at no extra cost in the LLL[ [] ). This quasielectron has a gap that remains nonzero in the thermodynamic limit. We have shown that it is given by $\approx 0.09 g$ where $g=\sqrt{32 \pi} \hbar \omega_{c} a_{s} / \ell_{z}$ and $\ell_{z}$ is the confinement length along the $z$-axis.

This nonzero gap also appears in the spectrum of collective density excitations. This is most clearly seen in the spherical geometry. The motion of the particles is restricted to a sphere which experiences uniform flux from its center, as created by a magnetic monopole. The flux $2 \mathrm{~S}$ in dimensionless units is quantized by Dirac's condition : it is a positive integer. The radius of the sphere is given by $R=\ell \sqrt{2 S}$ where $\ell$ is the magnetic length. The problem of Landau 


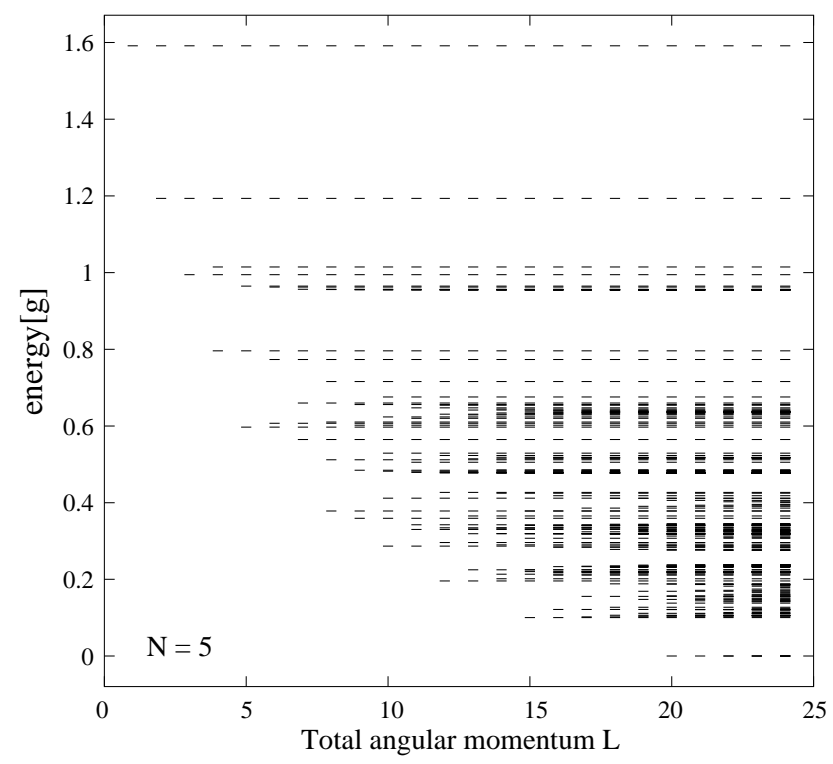

Figure 1: Spectrum of $\mathrm{N}=5$ bosons in the unbounded disk geometry. The Laughlin state is at zero-energy and $\mathrm{L}_{z}=20$.

levels on the sphere was solved long ago by Tamm [17]. The eigenstates are monopole harmonics, a generalization of spherical harmonics 18, 19, 20]. The LLL has $2 \mathrm{~S}+1$ states, the well-known Landau degeneracy. Wavefunctions constructed in the disk geometry may be translated on the sphere by a stereographic projection [20]. It is important to note that in general quantum Hall fluids require a finite shift on the sphere : the relation between the flux and the number of particle is of the form $2 S=(N / \nu)-X$ and the quantity $X$ may be computed once we have a guess for the wavefunction. In the case of the Laughlin wavefunction, we have $2 S=m(N-1)$. Hierarchical constructions give us definite predictions about the shift that can be then tested by exact diagonalization. The spherical geometry preserves the full SU(2) symmetry and thus the eigenstates are classified by their total angular momentum. Here the Laughlin state is a singlet state of the total angular momentum. It is separated by a clear gap from a branch of isolated states that abruptly terminates at $L=N$ : this is what we expect for two-particle states made of quasiparticles each having angular momentum $L=N / 2$.

\section{B. other fractions}

The standard hierarchies may be easily translated to the case of Bose particles. It is natural to expect that the Bose analog of the prominent Jain sequence of fractions 21] should display the most stable states. This sequence is realized when $\nu=p /(p \pm 1)$. These states are naturally "explained" in the composite fermion scheme : each boson captures a vortex with one quantum of statistical flux and is transmuted into a fermionic particle, the composite fermion $(\mathrm{CF})$. In a mean-field picture, the fraction $\nu=p /(p \pm 1)$ is realized when the CFs occupy exactly $p$ Landau levels. On the sphere we find that there are incompressible states along the lines given by :

$$
2 S=\frac{p \pm 1}{p} N \mp p-1 \quad \text { for } \quad \nu=\frac{p}{p \pm 1} .
$$

In fig.(2) we have displayed the corresponding candidates. In the interval $1>\nu>1 / 2$, we observe candidate states for $\nu=2 / 3,3 / 4,4 / 5$. They have the expected scaling properties towards the thermodynamic limit [15]. These fluids display also a collective mode which is gapped for all values of the angular momentum as is the case of the parent $\nu=1 / 2$ fluid. The apparition of these fractions follows the standard hierarchical scheme : deviation from the commensurability in Eq.(7) leads to the creation of extra quasiparticles that finally condense in a new incompressible fluid. 


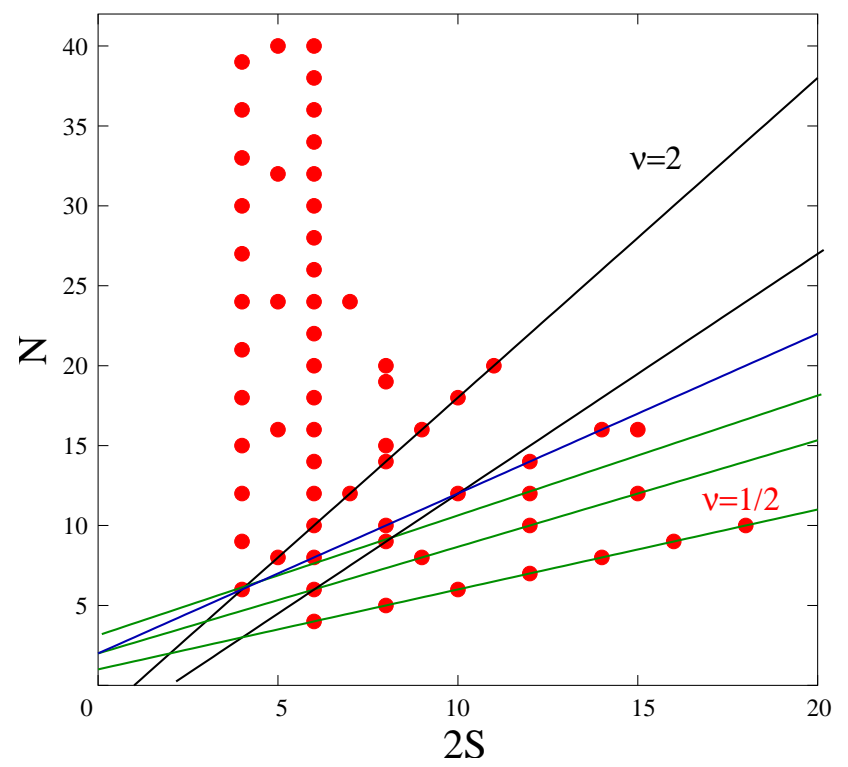

Figure 2: Candidates for incompressible fluids in the plane number of bosons vs flux quanta in the spherical geometry. From bottom to top, we have the line $\nu=1 / 2$ then $\nu=2 / 3,3 / 4$ then the $\nu=1$ states with the Pfaffian shift and finally the hierarchical states at $\nu=3 / 2,2$.

\section{THE PAIRED $\nu=1$ STATE}

In the Jain principal sequence of fractions there is an accumulation point at $\nu=1$ at which the CFs feel zero net flux. This is the Bose analog of the $\nu=1 / 2$ state for fermions. We thus expect that either there is some kind of Fermi sea of composite fermions as is the case for electrons with Coulomb repulsion or it may also happen that this Fermi sea is unstable towards pairing of CFs [22, 23]. This latter case would be the Bose analog of the paired state suggested to exist at the enigmatic $\nu=5 / 2$ state of electrons. On the sphere, the zero-flux on the CFs is realized for $2 S=N-1$. When $\mathrm{N}$ is a square, the CFs fill a closed shell and there are low-lying excitations that have a characteristic structure [24, 25]. One expects that between the closed-shell states the angular momentum of the ground state is determined by the second Hund's rule (max L). While this law applies for fermions with Coulomb or hard-core interaction, there are deviations in the case of bosons. In addition there is no clear scaling to the infinite system for the ground state energy. This can be taken as evidence that the Fermi sea is unstable in the Bose case. A prototypical wavefunction describing the pairing of the CFs is the so-called Pfaffian :

$$
\Psi=\operatorname{Pf}\left\{\frac{1}{u_{i} v_{j}-u_{j} v_{i}}\right\} \prod_{i<j}\left(u_{i} v_{j}-u_{j} v_{i}\right)^{q} .
$$

The symbol Pf stands for the Pfaffian and $N$ should be even and we have defined $u=\cos (\theta / 2) \mathrm{e}^{i \varphi / 2}, v=\sin (\theta / 2) \mathrm{e}^{-i \varphi / 2}$. Given an antisymmetric $N \times N$ matrix $A_{i j}$ it is defined by :

$$
\operatorname{Pf}\left\{A_{i j}\right\}=\sum_{\sigma} \epsilon_{\sigma} A_{\sigma(1) \sigma(2)} \ldots A_{\sigma(N-1) \sigma(N)},
$$

where $\sigma$ are permutations of the index with $N$ values. For $q=1$ the wavefunction Eq. (88) describes bosons at filling factor $\nu=1$ while for $q=2$ it describes a fermion state at $\nu=1 / 2$. The filling factor is $\nu=1 / q$ and this wavefunction will fit on the sphere if we have :

$$
2 S=q(N-1)-1 .
$$

In the Bose case, there is a complete series of incompressible states at the locations predicted by this equation. The gap extrapolates to a nonzero value in the thermodynamic limit. In addition, when one add or remove one flux quantum from the perfect matching condition one observes the creation of two-particles states contrary to the case of the (unpaired) Laughlin fluids. This is a direct spectroscopic evidence for the paired nature of the fluid at $\nu=1$. 


\section{INCOMPRESSIBILITY AT $\nu>1$}

For bosons it is possible to stay entirely into the LLL while reaching filling factors greater than 1 . The Jain sequence extends naturally in this domain for $\nu=p /(p-1)$ and there are states with spectra displaying the characteristics of incompressibility on the lines predicted by Eq.(17). However they show very irregular behavior as a function of the number of particles. This novelty with respect to fermions lead to incompressible states that may be the clustered Read-Rezayi states [26]. It has been pointed out in the torus geometry that there are fractions for $\nu=k / 2$ where $k$ is an integer [13]. These states have a ground state degeneracy which is exactly that expected for the Read-Rezayi states and they have also high overlap with the corresponding trial wavefunctions.The situation is not so clear in the spherical geometry. For even higher filling factor there should be a quantum phase transition towards the vortex lattice [13, 27] in the region $\nu \approx 6$.

\section{ROTATING FERMIONS}

It has been pointed out recently [] that a single species of hyperfine fermions interacting through $p$-wave scattering leads exactly to the hard-core model with only one nonzero pseudopotential when projected onto the LLL. So the celebrated Laughlin wavefunction for fermions is now the exact ground state of the system for the filling $1 / 3$. It is known that the hard-core model displays FQHE properties that are close to those of the Coulomb case. Here the principal sequence has gaps whose order of magnitude is ruled by the combination :

$$
g_{f}=\sqrt{\frac{2}{\pi}} \frac{\hbar^{2}}{m} \frac{a_{p}^{3}}{\ell_{z} \ell^{4}} .
$$

Gaps were estimated for the principal sequence and if one is able to enhance the $p$-wave scattering length up to values comparable to the $s$-wave case then the FQHE for Fermi gases will have a strength comparable to the Bose case. Concerning the fraction $\nu=1 / 3$ one should note that it has charged excitations that are gapped (quasielectrons) as well as gapless (quasiholes), similarly to the Bose case. At exactly half-filling, there is a Fermi sea of two-flux CFs. It appears on the sphere when the flux is given by $2 S=2(N-1)$. Using the model of free CFs one can extract the effective mass of the CFs by considering the excitations above the closed shell configurations that occur when $\mathrm{N}$ is a perfect square. Finally we note that it has been recently suggested that the FQHE should also appear in Fermi gases with dipolar interactions [28]. If the interaction has a $1 / r^{3}$ law then it is intermediate between the pure hard-core forces that we have discussed so far and the Coulomb interaction relevant to electrons in semiconductor heterostructures. There is scattering in all allowed partial waves (all pseudopotentials are nonzero) hence the Laughlin state is no longer an exact eigenstate but it will remain an excellent approximation. Quasiholes are now gapped as is the case for the Coulomb interaction. For small filling factor, it is likely that the Laughlin liquids will compete with the Wigner crystal state. This is a very interesting situation since this crystal, if realized, would be free of disorder and hence amenable to detailed experimental and theoretical studies.

\section{CONCLUSIONS}

Trapped gases offer a so far theoretical opportunity to study the physics of the FQHE in new conditions. The Bose gases display a new version of the principal sequence of FQHE fractions and also paired states that have so far no equivalent in the electronic world. The elusive fractional statistics may be eventually observed experimentally [29] The Fermi gases may be a perfect incarnation of the hard-core model of the FQHE, displaying notably the elusive Fermi sea of CFs at half-filling. With dipolar fermions one may even study the competition with crystalline states at low filling factor of the LLL.

We thank Yvan Castin and Jean Dalibard for numerous discussions. Some of the numerical calculations have been performed thanks to a computer time allocation of IDRIS-CNRS.

[1] M. R. Matthews, B. P. Anderson, P. C. Haljan , D. S. Hall, C. E. Wieman, and E. A. Cornell, Phys. Rev. Lett. 83, 2498 (1999).

[2] K. W. Madison , F. Chevy, W. Wohlleben, and J. Dalibard, Phys. Rev. Lett. 84, 806 (2000); F. Chevy, K. Madison, and J. Dalibard, Phys. Rev. Lett. 85, 2223 (2000)

[3] J. R. Abo-Shaeer, C. Raman, J. M. Vogels, and W. Ketterle, Science 292, 476 (2001). 
[4] P. C. Haljan , I. Coddington, P. Engels, and E. A. Cornell, Phys. Rev. Lett. 87, 210403 (2001).

[5] N. K. Wilkin, J. M. F. Gunn, and R. A. Smith, Phys. Rev. Lett. 80, 2265 (1998).

[6] M. Cozzini, S. Stringari, V. Bretin, P. Rosenbusch, and J. Dalibard, Phys. Rev. A67, 021602 (2003).

[7] N. Regnault and Th. Jolicoeur, e-print cond-mat/0404093

[8] S. Trugman and S. Kivelson, Phys. Rev. B59, 8084 (1985).

[9] P. Rosenbusch, D. S. Petrov, S. Sinha, F. Chevy, V. Bretin, Y. Castin, G. Shlyapnikov, and J. Dalibard, Phys. Rev. Lett. 88, 250403 (2002).

[10] C. A. Regal, C. Ticknor, J. L. Bohn, and D. S. Jin, Phys. Rev. Lett. 90, 053201 (2003).

[11] N. R. Cooper and N. K. Wilkin, Phys. Rev. B60, R16279 (1999).

[12] N. K. Wilkin and J. M. F. Gunn , Phys. Rev. Lett. 84, 6 (2000).

[13] N. R. Cooper, N. K. Wilkin, and J. M. F. Gunn, Phys. Rev. Lett. 87, 120405 (2001).

[14] N. Regnault and Th. Jolicoeur, Phys. Rev. Lett. 91, 030402 (2003).

[15] N. Regnault and Th. Jolicoeur, Phys. Rev. B, in press (2004).

[16] F. D. M. Haldane and E. H. Rezayi, Phys. Rev. Lett. 54, 237 (1985).

[17] I. Tamm, Z. Phys. 71, 141 (1931).

[18] T. T. Wu and C. N. Yang, Nucl. Phys. B107, 365 (1976); Phys. Rev. D16, 1018 (1977).

[19] F. D. M. Haldane, Phys. Rev. Lett. 51, 605 (1983).

[20] G. Fano, F. Ortolani, and E. Colombo, Phys. Rev. B34, 2670 (1986).

[21] J. K. Jain, Phys. Rev. Lett. 63, 199 (1989).

[22] G. Moore and N. Read, Nucl. Phys. B360, 362 (1991).

[23] M. Greiter, X. G. Wen, and F. Wilczek, Phys. Rev. Lett. 66, 3205 (1991); Nucl. Phys. B374, 567 (1992).

[24] E. Rezayi and N. Read, Phys. Rev. Lett. 72, 900 (1994).

[25] R. Morf and N. d'Ambrumenil, Phys. Rev. Lett. 74, 5116 (1995).

[26] N. Read and E. H. Rezayi, Phys. Rev. B54, 16864 (1996); Phys. Rev. B59, 8084 (1999).

[27] J. Sinova, C. B. Hanna, and A. H. MacDonald, Phys. Rev. Lett. 89, 030403 (2002).

[28] M. A. Baranov, K. Osterloh, and M. Lewenstein, e-print cond-mat/0404329

[29] B. Paredes, P. Fedichev, J. I. Cirac, and P. Zoller, Phys. Rev. Lett.87, 010402 (2001); B. Paredes, P. Zoller, and J. I. Cirac, Phys. Rev. A 66, 033609 (2002). 